\title{
Deep brain stimulation procedure complicated by spontaneously resolved pneumothorax in a patient with cervical dystonia
}

\author{
Michał Sobstyl $^{1}$, Angelika Stapińska-Syniec ${ }^{1}$, Marcin Rylski ${ }^{2,3}$ \\ ${ }^{1}$ Department of Neurosurgery, Institute of Psychiatry and Neurology, Warsaw, Poland \\ ${ }^{2}$ Department of Neuroradiology, Institute of Psychiatry and Neurology, Warsaw, Poland \\ ${ }^{3}$ Department of Clinical Cytology, Centre of Postgraduate Medical Education, Warsaw, Poland
}

Key words: deep brain stimulation, cervical dystonia, pneumothorax, procedure-related complications

(Neurol Neurochir Pol 2021; 55 (1): 110-112)

\section{To the Editors:}

Deep brain stimulation (DBS) is an established and effective treatment modality for patients suffering from movement disorders such as Parkinson's Disease, dystonia, and essential tremor [1-4]. The most life-threatening complication of DBS is an intracerebral haemorrhage (ICH). However, vascular injury or lung apex injury in the neck and upper thoracic regions has not been reported among possible DBS procedure-related complications.

Herein, we report the first case of subcutaneous pneumothorax confirmed by a plain chest X-ray with neck and chest computed tomography after the placement of an extension during the internalisation of further DBS hardware components.

A 58-year-old right-handed male with a 15-year history of cervical dystonia (CD) was admitted to the neurosurgical department for a scheduled second right-side staged implantation of a DBS system. His medical history was unremarkable apart from the treatment of CD with pharmacotherapy and botulinum toxin injections. The stereotactic target was chosen as a posteroventrolateral part of the globus pallidus internus (GPi). The first left-sided GPi DBS procedure was uneventful. The second right-sided DBS lead implantation was also uneventful. Subsequently, the right IPG ACTIVA 37603 was placed in a subclavicular region and connected to a DBS electrode by an extension.

During the tunnelling, rapid ceasing of subcutaneous tissue resistance resulted in pushing the tip under the right collarbone. The tunnelling rod was moved backwards and forwards again to the extent of a pocket made for an IPG. Before the extubation, a right-sided subcutaneous pneumothorax with typical crackling was noted. An immediate plain X-ray of his chest revealed that the pneumothorax was affecting the right lung apex (Fig. 1A) and subcutaneous soft tissue. The thoracic surgeon consulted the patient. There was no indication for surgical insertion of pleural drainage. The patient was monitored in an intensive care unit for three days. A repeated X-ray on postoperative day 2 revealed no enlargement of the pneumothorax. On postoperative day 5, a neck and thoracic CT was conducted to assess the pneumothorax (Fig. 1B-H). The neck computed tomography enabled the location of the right extension compared to the left extension (Fig. 1B-D). There was a marked resolution of the air in the neck and upper thoracic regions. Only small air bubbles could be recognised on the chest computed tomography examination at that time (Fig. 1E-H). The subcutaneous pneumothorax was not palpable on postoperative day 5 . The right IPG was turned on. The patient was discharged on postoperative day 7 without any neurological sequelae and followed up at scheduled postoperative visits at three, six, 12 , and 18 months. The patient responded well to bilateral GPi DBS, maintaining good symptoms improvement over 18 months without any complications.

We here present, to the best of our knowledge, the first case of subcutaneous pneumothorax immediately after a DBS procedure due to a lung apex injury caused by a tunnelling rod, with detailed confirmation on radiological examinations. The standard tunnelling tool accessory kit includes one tunnelling 


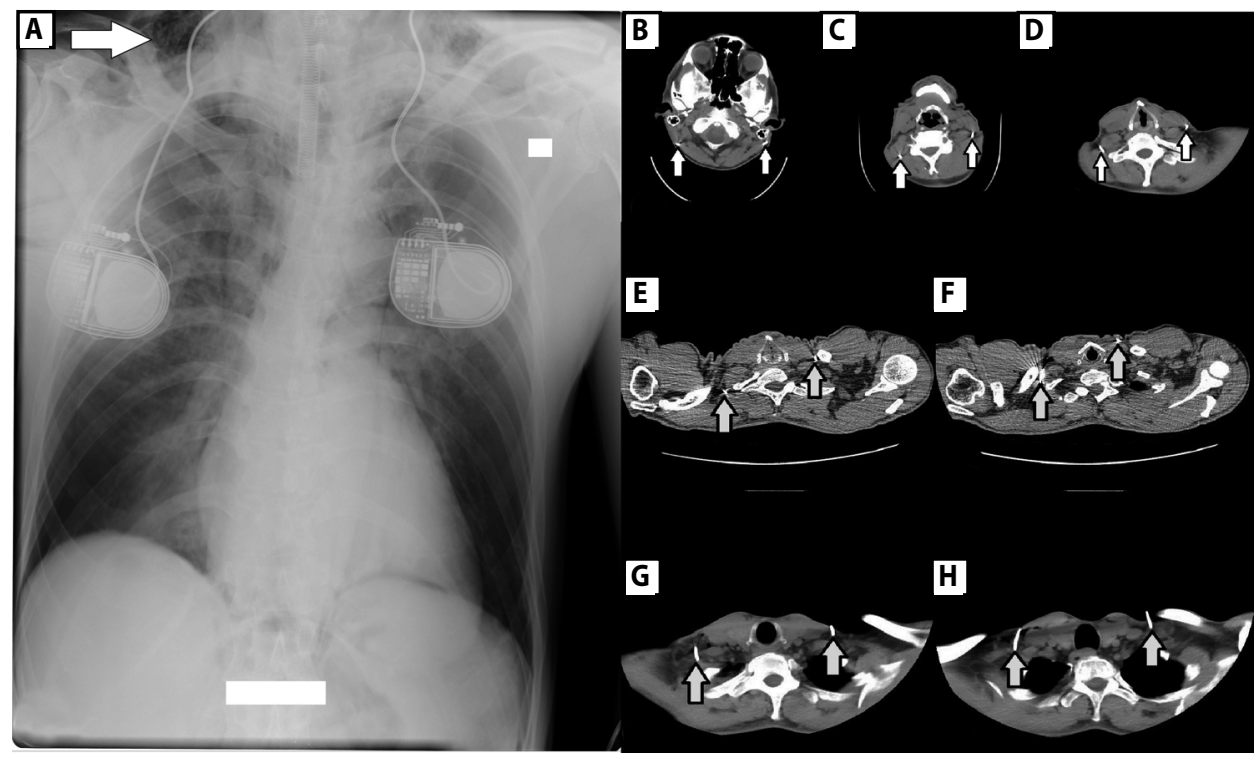

Figure 1. A. Plain X-ray of chest taken immediately after implantation of right deep brain stimulation system. White arrow shows air in subcutaneous tissue and region of right lung apex; B-H. Computed tomography of neck (CT) in axial planes: B. CT image showing course of connectors with white arrows at level of mastoid bones bilaterally; C. CT image showing right connector running in perivertebral space while left connector is located just lateral and posterior to left sternocleidomastoid muscle; D. CT image indicating course of right connector posterior to common carotid artery while left connector is placed in correct position lateral to left sternocleidomastoid muscle. Computed tomography of chest (CT) in axial planes; E. CT image showing course of connectors with white arrows at level of lower neck. Right connector lies posterior to common carotid artery. Subcutaneous pneumothorax is recognised just lateral to right connector; F. CT image indicating location of right and left connectors. Right connector courses upwards before right clavicle. Air bubbles appear in soft tissue of neck and upper chest on right side; G, H. CT images in axial planes depicting further course of both connectors in upper chest. Pneumothorax is visible only on right side (air bubbles in soft tissue around right connector)

rod with a handle and three tips: a single tip, a dual tip, and a wedge tip. The three threaded metal tips used for tunnelling are designed to create a tunnel to allow the passage of a single carrier (single tip) or for the passage of a dual carrier (dual tip and wedge tip).

For our tunnelling we used a dual tip, which is more rounded than a single tip. We avoided using tunnelling rods with single or wedge tips. In our opinion, the single tip allows poorer control when resistance suddenly ceases and the force put on the tunnelling handle can push the tip forward in a way that is more difficult to control. Wedge tips are sharper and can more easily cause soft tissue injury or even vascular injury. Tunnelling is more hazardous in slim patients who have less subcutaneous fat tissue. Even slightly uncontrolled pushing and introduction of the tunnelling tip under the collarbone can injure the lung apex with subsequent development of a subcutaneous pneumothorax, as in the described patient. This rare complication proved to be self-limiting, and the pneumothorax resolved within seven days without any sequel. In our patient, the neck CT showed very clearly that the right connection cable was tunnelled within the proximity, just posterior to the right internal carotid artery.

We advise that in slim patients with long necks the tunnelling of an extension should be performed more superficially, directly under the skin rather than in deeper layers. Tunnelling too deeply can increase vascular injury in slender patients.

Analysing the DBS literature regarding adverse events, we were able to find one patient with an adverse event listed as pneumothorax who underwent bilateral implantation of DBS electrodes in the anterior limb of the internal capsule for the treatment of chronic refractory obsessive-compulsive disorder [5]. All serious adverse events, including the deaths related to DBS procedures, are caused by intracerebral haemorrhagic complications.

Although DBS procedures are a standard treatment for movement disorders, these possible serious complications have not been previously reported. No deaths or acute injury to vascular structures have been described due to tunnelling and the passage of an extension.

\section{References}

1. Hariz MI, Rehncrona S, Quinn NP, et al. Multicentre Advanced Parkinson's Disease Deep Brain Stimulation Group. Multicenter study on deep brain stimulation in Parkinson's disease: an independent assessment of reported adverse events at 4 years. Mov Disord. 2008; 23(3): 416-421, doi: 10.1002/mds.21888, indexed in Pubmed: 18067188. 
2. Isaias IU, Alterman RL, Tagliati M. Deep brain stimulation for primary generalized dystonia: Iong-term outcomes. Arch Neurol. 2009; 66(4): 465-470, doi: 10.1001/archneurol.2009.20, indexed in Pubmed: 19364931.

3. Larson PS. Deep brain stimulation for movement disorders. Neurotherapeutics. 2014; 11(3): 465-474, doi: 10.1007/s13311-0140274-1, indexed in Pubmed: 24833244.
4. Lyons KE, Pahwa R. Deep brain stimulation and essential tremor. J Clin Neurophysiol. 2004; 21(1): 2-5, doi: 10.1097/00004691200401000-00002, indexed in Pubmed: 15097289.

5. Menchón JM, Real E, Alonso P, et al. A prospective international multi-center study on safety and efficacy of deep brain stimulation for resistant obsessive-compulsive disorder. Mol Psychiatry. 2019 [Epub ahead of print], doi: 10.1038/s41380-019-0562-6, indexed in Pubmed: 31664175. 